Article

\title{
Integrative Analysis of Dysregulated lncRNA-Associated ceRNA Network Reveals Functional lncRNAs in Gastric Cancer
}

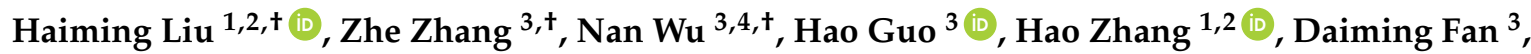 \\ Yongzhan $\mathrm{Nie}^{3, *}$ and Yuanning Liu ${ }^{1,2, * \text { ib }}$ \\ 1 College of Computer Science and Technology, Jilin University, Changchun 130012, Jilin, China; \\ liuhaiming0702@163.com (H.L.); zhangh@jlu.edu.cn (H.Z.) \\ 2 Key Laboratory of Symbolic Computation and Knowledge Engineering, Ministry of Education, Jilin \\ University, Changchun 130012, Jilin, China \\ 3 State Key Laboratory of Cancer Biology, National Clinical Research Center for Digestive Diseases, Xijing \\ Hospital of Digestive Diseases, Fourth Military Medical University, Xi'an 710032, Shaanxi, China; \\ zz141421@126.com (Z.Z.); 13119110590@136.com (N.W.); h.guo@foxmail.com (H.G.); \\ fandaim@fmmu.edu.cn (D.F.) \\ 4 College of Life Sciences, Northwest University, Xi'an 710032, Shaanxi, China \\ * $\quad$ Correspondence: liuyn@jlu.edu.cn (Y.L.); yongznie@fmmu.edu.cn (Y.N.) \\ + These authors contributed equally to this work.
}

Received: 3 May 2018; Accepted: 12 June 2018; Published: 18 June 2018

\begin{abstract}
Mounting evidence suggests that long noncoding RNAs (lncRNAs) play important roles in the regulation of gene expression by acting as competing endogenous RNA (ceRNA). However, the regulatory mechanisms of IncRNA as ceRNA in gastric cancer (GC) are not fully understood. Here, we first constructed a dysregulated lncRNA-associated ceRNA network by integrating analysis of gene expression profiles of lncRNAs, microRNAs (miRNAs), and messenger RNAs (mRNAs). Then, we determined three lncRNAs (RP5-1120P11, DLEU2, and DDX11-AS1) as hub lncRNAs, in which associated ceRNA subnetworks were involved in cell cycle-related processes and cancer-related pathways. Furthermore, we confirmed that the two lncRNAs (DLEU2 and DDX11-AS1) were significantly upregulated in GC tissues, promote GC cell proliferation, and negatively regulate miRNA expression, respectively. The hub lncRNAs (DLEU2 and DDX11-AS1) could have oncogenic functions, and act as potential ceRNAs to sponge miRNA. Our findings not only provide novel insights on ceRNA regulation in GC, but can also provide opportunities for the functional characterization of lncRNAs in future studies.
\end{abstract}

Keywords: competing endogenous RNA; long non-coding RNA; gastric cancer; network analysis; DLEU2; DDX11-AS1

\section{Introduction}

Gastric cancer (GC) is one of the leading causes of cancer-related death worldwide, and imposes a considerable global health burden [1,2]. In 2012, an estimated 723,100 deaths from GC and nearly half of the burden occurred in Eastern Asia, particularly in China, Korea, and Japan [3]. In China, GC was the second most common cancer, as reported by the National Central Cancer Registry (NCCR) [4].

Previous studies have demonstrated that coding RNAs and non-coding RNAs (ncRNAs) are closely related to cancer [5]. MicroRNAs (miRNAs) are an important subclass of endogenous non-coding RNAs with a typical length of 23 nucleotides [6], and play crucial roles in cancer by regulating gene expression at the post-transcriptional level [7]. Long non-coding RNAs (lncRNAs), 
another important subclass of endogenous non-coding RNAs, are longer than 200 nucleotides, and lack protein-coding capability [8]. Similar to coding RNAs and miRNAs, some lncRNAs can drive cancer phenotypes [9]. However, the complete spectrum of mechanisms by which lncRNAs affect cancer development and pathology remains to be fully elucidated.

Recent studies have demonstrated that lncRNA can act as a miRNA sponge via competing endogenous RNA (ceRNA) activity $[10,11]$. Understanding this novel RNA crosstalk will lead to significant insights into gene regulatory networks and have implications in human development and disease [12]. For example, PTENP1, a pseudogene of the tumor-suppressor gene PTEN, is among the first reported miRNA sponges with a function in cancer [13]. BRAF is an oncogene and its overexpression leads to enhanced activity of the MAPK signaling pathway [14]. Overexpression of the BRAF pseudogene results in upregulation of BRAF. HOTAIR acts as an miRNA sponge to regulate HER2 expression by sponging hsa-miR-331-3p in GC [15]. GAPLINC acts as a ceRNA for hsa-miR-211-3p, and mediates cell migration and proliferation in GC [16]. KRTAP5-AS1 and TUBB2A participate in the regulatory network of CLDN4 based on ceRNA activity in GC [17]. MEG3 can acts as a ceRNA to regulate GC progression [18].

Based on the ceRNA hypothesis, comprehensive analyses of IncRNA-associated ceRNA networks have been reported in ovarian cancer [19], glioblastoma [20], prostate cancer [10], thyroid carcinoma [21], and breast cancer [22]. Xia et al. [23] constructed a cancer-associated ceRNA network in GC based on lncRNA microarray data. Li et al. [24] showed the GC-specific lncRNA expression patterns, and constructed a ceRNA network based on The Cancer Genome Atlas (TCGA). Although previous studies have identified ceRNAs in GC, the functional roles and regulatory mechanisms of lncRNAs as ceRNAs in GC are not fully understood.

In this study, we first systematically integrated gene expression profiles of GC, miRNA target interactions, and significantly co-expressed gene pairs based on the ceRNA hypothesis. We then constructed dysregulated lncRNA-associated ceRNA network. After the network topological analysis, we determined that the hub lncRNAs play crucial roles in GC development. By reconstructing and functionally analyzing the hub lncRNA-associated subnetworks, we identified three hub IncRNAs (RP5-1120P11, DLEU2, and DDX11-AS1) that are involved in cell cycle-related processes and cancer-related pathways. Finally, we validated that DLEU2 and DDX11-AS1 are significantly upregulated in GC tissues by using qRT-PCR. Cellular experimental results indicated that the two lncRNAs promote GC cell proliferation and negatively regulate miRNA expression. Through an integrative analysis of dysregulated lncRNA-associated ceRNA network (Figure S1), we determined that hub lncRNAs (DLEU2 and DDX11-AS1) have oncogenic functions, and act as potential ceRNAs to sponge miRNA. Our findings can be used to reveal ceRNA regulation in GC, and provide novel insights on functional characterization of lncRNAs.

\section{Materials and Methods}

\subsection{GDC Data Collection and Processing}

Gastric cancer gene expression data were obtained from Genomic Data Commons (GDC, https: // cancergenome.nih.gov/) [25]. The upper quantile normalized FPKM (fragments per kilobase per million) values and the normalized count in reads-per-million-miRNA-mapped values were used. Patients with follow-up time exceeding three years were excluded. Overall, a total of 364 samples, including 337 GC samples and 27 normal samples, were included in our study and each sample consisted of the corresponding RNA-seq data and miRNAseq data.

To assemble a lncRNA-associated ceRNA data, we downloaded the RNA gene annotation and sequence data from GENCODE v26 (https: / / www.gencodegenes.org/), which aims to identify all gene features in the human genome using a combination of computational analysis, manual annotation, and experimental validation [26]. Transcripts longer than $200 \mathrm{nt}$ and categorized as "lincRNA", "antisense", "processed_transcript", and "non_coding" were identified as lncRNAs. Genes categorized 
as "protein_coding" were considered messenger RNAs (mRNAs). In total, 19,799 mRNAs and 13,545 lncRNAs in 364 samples were annotated

Genes expressed in more than $90 \%$ of samples were retained, and the values of zero or NA in the expression profile were replaced with the (minimum non-zero FPKM value) $/ 2$ of the corresponding genes. All the expression profile values were then $\log 2$ transformed. Therefore, the expression profiles of 16,363 mRNAs, 440 miRNAs, and 4211 lncRNAs in 364 samples were selected for further analysis.

\subsection{Identifying Differentially Expressed $m R N A s$, IncRNAs, and miRNAs Related to GC}

To identify differentially expressed lncRNAs (DELs), mRNAs (DEMs), and miRNAs (DEMis), we used the two-tailed Mann-Whitney $U$ test to compare the expression values in cancer with those in normal. For all $p$-values, FDR (false discovery rate) was used to correct the statistical significance of multiple testing by the Benjamini-Hochberg method. In this paper, we mainly focused on the upregulated lncRNAs, upregulated mRNAs, and downregulated miRNAs, which could have oncogenic functions based on ceRNA activity. Therefore, we defined differentially expressed lncRNAs/mRNAs with fold change $\geq 1$ and adjusted $p$-value $<0.001$ as DELs/DEMs. In order to avoid insufficient number of downregulated miRNAs, we defined differentially expressed miRNAs with fold change $\leq-0.6$ and adjusted $p$-value $<0.001$ as DEMis.

\subsection{Identification of miRNA-Target Interactions}

MicroRNA sequences were obtained from the TargetScan website (http:/ / www.targetscan.org/) [27]. LncRNA sequences were obtained from the GENCODE v26. miRNA-mRNA interactions were obtained from three reliable online miRNA-target databases: miRWalk 2.0 [28], miRTarBase 6.0 [29], and DIANA-TarBase v7.0 [30]. miRNA-IncRNA interactions were predicted using TargetScan 7.0 [27] with default parameters. After integrating the above methods, a total of 148,459 non-redundant reliable miRNA-mRNA interactions and 29,523 miRNA-lncRNA predicted interactions were obtained. We then selected 1146 reliable DEM-DEMi interactions and 2794 predicted DEL-DEMi interactions for further analysis.

\subsection{Identification of Potential ceRNA Triples Based on Gene Expression}

The competition of lncRNAs with mRNAs to sponge miRNAs is reflected at the gene expression level [31]. These lncRNA-miRNA-mRNA interactions were defined as ceRNA triples, and the potential ceRNA triples were identified as follows: (1) expression correlations among all mRNAs, IncRNAs, and miRNAs in GC were evaluated by the Pearson correlation coefficient (PCC). The PCC of gene pair $(\mathrm{X}$ and $\mathrm{Y})$ is defined as

$$
\operatorname{PCC}(X, Y)=\frac{1}{n-1} \sum_{i=1}^{n}\left(\frac{\operatorname{Exp}(X, i)-\overline{\operatorname{Exp}}(X)}{\sigma(X)}\right) \cdot\left(\frac{\operatorname{Exp}(Y, i)-\overline{\operatorname{Exp}}(Y)}{\sigma(Y)}\right),
$$

where $n$ is the number of GC cancer samples; $\operatorname{Exp}(X, i)(\operatorname{Exp}(Y, i))$ is the expression level of $X$ $(Y)$ in sample $i ; \overline{\operatorname{Exp}}(X)(\operatorname{Exp}(Y, i))$ denotes the average expression level of $X(Y)$, and $\sigma(X)(\sigma(Y))$ represents the standard deviation of expression level of $X(Y)$. In order to obtain the distribution of the overall PCC in GC, we calculated the PCC between 16,363 mRNAs, 440 miRNAs, and 4211 lncRNAs, respectively. We then obtained the PCC of 68,904,593 mRNA-lncRNA pairs, $1,852,840$ lncRNA-miRNA pairs, and 7,199,720 mRNA-miRNA pairs. (2) To obtain the significant co-expressed pairs, PCC higher than the threshold of $95 \%$ was considered a significant positively co-expressed pair, and lower than the threshold of $95 \%$ was considered as a negatively co-expressed pair. Therefore, DEL-DEM pairs with PCC $>0.292$ and $p$-value $<0.0001$ was considered as significantly co-expressed DEL-DEM pairs (Figure S2A). DEM-DEMi pairs with PCC $<-0.275$ and $p$-value $<0.0001$ were considered as significantly negatively co-expressed DEM-DEMi pairs (Figure S2B). DEL-DEMi pairs with PCC $<-0.233$ and $p$-value $<0.0001$ were considered as significantly negatively DEL-DEMi 
pairs (Figure S2C). (3) For a given significant co-expressed DEM-DEL pair, both DEM and DEL interacted with the same DEMi, and were negatively co-expressed with the same DEMi, and this DEL-DEMi-DEM interaction was defined as a potential ceRNA triple.

\subsection{Construction Network and Topological Analysis}

To clarify the roles of the dysregulated lncRNA-associated ceRNA network, we constructed a co-expression network of DEL-DEMi-DEM interactions based on the potential ceRNA triples, and visualized this ceRNA network by Cytoscape 3.5.1 [32]. Betweenness centrality (BC) is a measure of centrality in a network based on the number of shortest paths from every node to all others passing through this node. In a network, a node with a higher BC is implied to act as a bridge to connect different nodes and control the network communications. In this study, BC was considered as the topological analysis measure to select the hub lncRNAs. The BC of a node $i$ is defined as

$$
\text { Betweenness centrality }=\sum_{s \neq i \neq t} \frac{p_{s t}(i)}{p_{s t}}
$$

where $p_{s t}$ is the total number of the shortest paths from node $s$ to node $t$, and $p_{s t}(i)$ is the number of these paths that pass through node $i$.

We assumed that the hub lncRNAs play critical roles in the ceRNA network. Accordingly, we reconstructed the hub lncRNA-associated subnetwork with the nodes, which is the hub lncRNA related to the first-neighbor miRNAs and the second-neighbor mRNAs in the ceRNA network.

\subsection{Functional Enrichment Analysis}

To reveal the function of IncRNA-associated ceRNA network, the DEMs were subjected to functional enrichment analysis via gene ontology (GO) and Kyoto Encyclopedia of Genes and Genomes (KEGG) with the R package cluster Profiler [33]. GO is a structured standard biological model established by the GO consortium, which aims to establish a standard system for computing the functions of genes, covering biological processes (BP), molecular functions (MF), and cellular components (CC) [34]. KEGG is widely used as a reference for integrating large-scale molecular datasets generated by sequencing and high-throughput experimental technologies [35]. In this study, we considered functional categories with a $p$-value $<0.05$.

\subsection{Patient Samples and GC Cell Lines}

Gastric cancer tissues and adjacent normal tissues were collected from patients who underwent surgery treatment at the Xijing Hospital (Xi'an, China). Written informed consents had been obtained from all patients who donated tissue samples, which was under the supervision of the Hospital's Protection of Human Subjects Committee. None of our study patients had received chemotherapy or radiotherapy before the surgery, and all tissues were paraffin-embedded after RNA and protein isolation. Human GC cell lines BGC823 and MKN45 were purchased from the Cell Bank of the Chinese Academy of Sciences. Cells were incubated at $37^{\circ} \mathrm{C}$ in a humidified atmosphere containing $5 \% \mathrm{CO}_{2}$ and cultured in Dulbecco's Modified Eagle's Medium (ThermoFisher Scientific, Waltham, MA, USA) combined with $10 \%$ fetal bovine serum (FBS).

\subsection{RNA Isolation and Real-Time PCR Analysis}

Total RNA was isolated using a Qiagen miRNeasy kit (Qiagen, Hilden, Germany) according to the manufacturer's protocol. Then, reverse transcription of RNA to cDNA was performed using the the Prime Script RT Master Mix (TaKaRa Bio Inc., Kusatsu, Japan). qRT-PCR was carried out using SYBR premix Ex Taq (Takara Bio Inc., Kusatsu, Japan). RNA qRT-PCR PrimerSets specifc for DLEU2, DDX11-AS1, hsa-miR-30-5p, and hsa-miR-145-5p were designed by RiboBio (Guangzhou, China). 
The expression levels of the RNA and internal control (U6) were measured using qRT-PCR (Bio-Rad, Hercules, CA, USA).

\subsection{RNA Interference by siRNA}

The siRNA targeting lncRNAs and siRNA control were synthesized by RiboBio (Guangzhou, China). The sequences of siRNAs were as follows: DLEU2-si1: 5'-CTAATAGCTTGAACCCTTT-3'; DLEU2-si2: 5'-CTAACTCCTCCACCAGAAA-3'; DDX11-AS1-si1: 5'-CTCATTCCTCTGCCTACAA-3'; DDX11-AS1-si2: 5'-AGACTCATGAGCTGAAGAT-3'. BGC823 and MKN45 cells cultured in 6-well plates were transfected with the siRNA according to the manufacturer's instructions. Cells were harvested at $36 \mathrm{~h}$ after transfection. The specific silencing of the two lncRNAs expression was assessed by using qRT-PCR.

\subsection{CCK-8 and Colony Formation Assays}

Cells were seeded in 96-well plates $\left(1 \times 10^{4}\right.$ cells per well), cultivated at $37^{\circ} \mathrm{C}$ in a $5 \% \mathrm{CO}_{2}$ humidified incubator for $24 \mathrm{~h}$. Ten microliters of Cell Counting Kit-8 solution (CCK-8) (Dojindo Laboratories, Kumamoto, Japan) were added to each well and incubated at $37{ }^{\circ} \mathrm{C}$ in a $5 \% \mathrm{CO}_{2}$ humidified incubator for $2 \mathrm{~h}$. Spectrometer Varioskan Flash (ThermoFisher Scientific) was used to measure the $450 \mathrm{~nm}$ absorbance. The CCK- 8 assay was performed every $24 \mathrm{~h}$ for 5 days. A proliferation curve was drawn with the time as the abscissa, and average absorbance value in each group as the ordinate. For the colony formation assay, Cells were seeded in 6-well plates $\left(1 \times 10^{3}\right.$ cells per well), and were incubated at $37^{\circ} \mathrm{C}$ in a $5 \% \mathrm{CO}_{2}$-humidified incubator. After 2 weeks, the cells were stained with Gentian violet (Beyotime Biotechnology, Shanghai, China). Triplicate reactions were performed for the experiment.

\subsection{Statistical Analysis of Biological Experimental Results}

Biological experimental results were analyzed using paired or unpaired $t$-test (GraphPad Software, La Jolla, CA, USA). Values of $p<0.05$ were considered statistically significant $\left({ }^{*}\right)$. ALL biological experimental results were presented as mean $\pm \mathrm{SD}$.

\section{Results}

\subsection{Identification of Differentially Expressed $\operatorname{lnc} R N A s, m R N A s$, and miRNAs}

We obtained the gene expression profiles of 337 GC samples and 27 normal samples from GDC and identified differentially expressed genes. A total of 757 lncRNAs, 2441 mRNAs, and 256 miRNAs were detected. Among them, 571 lncRNAs, 1097 mRNAs, and 227 miRNAs were significantly upregulated, while 186 lncRNAs, 1344 mRNAs, and 29 miRNAs were significantly downregulated in GC (Table S1). In this paper, we mainly focused on the upregulated lncRNAs, upregulated mRNAs, and downregulated miRNAs, which could have oncogenic functions based on ceRNA activity. Therefore, we selected 571 DELs, 1097 DEMs, and 29 DEMis from the significantly differentially expressed set for further analysis. To determine if the DELs, DEMs, and DEMis can be used to separate normal samples from cancer samples, we utilized "ward.D" algorithm and Euclidean distance to cluster these genes with the R package pheatmap. The heatmap indicated that all samples could be clearly separated into two groups, and less than $3 \%$ of the cancer samples were incorrectly classified (Figure 1). These differentially expressed genes were therefore considered as potential key regulators to construct the ceRNA network in GC. 


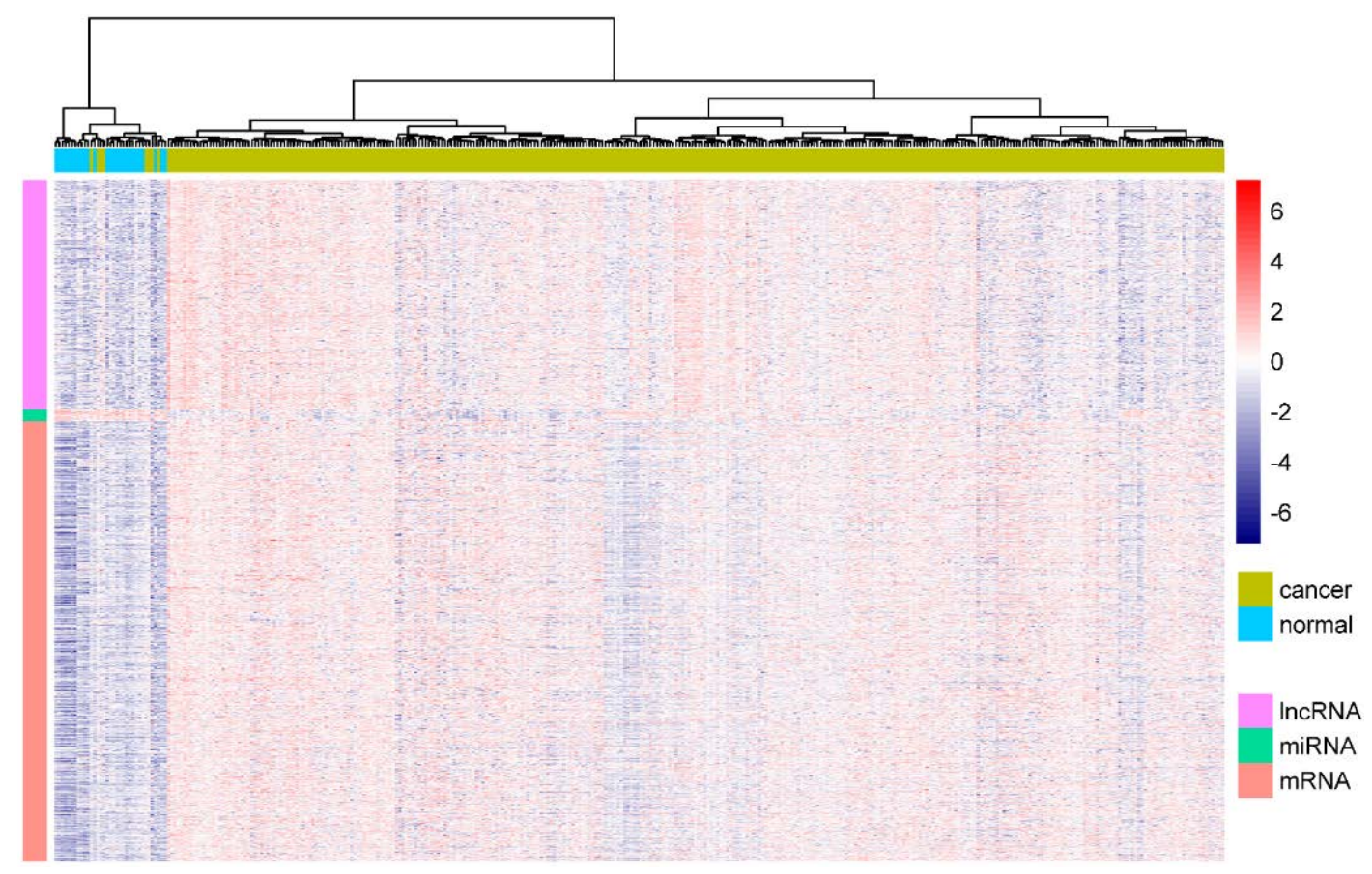

Figure 1. The heatmap of lncRNAs (DELs), mRNAs (DEMs), and miRNAs (DEMis) in gastric cancer (GC). The samples were represented in columns, and the genes were represented in rows, with different colors. The expression value for each row was normalized by the z-score. Red indicates high relative expression and blue indicates low expression of genes as shown in the scale bar.

\subsection{Construction and Functional Analysis of ceRNA Network in GC}

To construct the ceRNA network in GC, we focused on the relationships among the DELs, DEMs and DEMis. First, we identified miRNA-target interactions by integrating reliable online databases and prediction software. We then selected 1146 reliable DEM-DEMi interactions from 148,459 miRNA-mRNA interactions and 2794 candidate DEL-DEMi interactions from 29,523 miRNA-lncRNA interactions for further analysis. To reduce false positives, we used the PCC to filter the significant co-expressed DEL-DEMi-DEM interactions. After filtering the interactions, a total of 442 potential ceRNA triples were identified (Table S2). Based on the potential ceRNA triples, we constructed and visualized a dysregulated lncRNA-associated ceRNA network by Cytoscape (Figure 2A). The ceRNA network was a fully connected network, which consisted of 174 nodes (including 28 DELs, 130 DEMs, and 16 DEMis) and 173 edges. Then, we utilized the DEMs to reveal the gene functions of the dysregulated lncRNA-associated ceRNA network via the functional enrichment analysis. The ceRNA function analysis revealed $250 \mathrm{GO}$ terms in BP and 12 KEGG pathways (Figure 2B,C, and Table S3). Cancer is characterized by aberrant cell cycle activity [36], and most of these GO terms are cell cycle process related in the ceRNA network. Among the KEGG pathways, cell cycle, p53 signaling pathway, Fanconi anemia pathway, mismatch repair, DNA replication, and platinum drug resistance are cancer-related pathways [36-40]. 

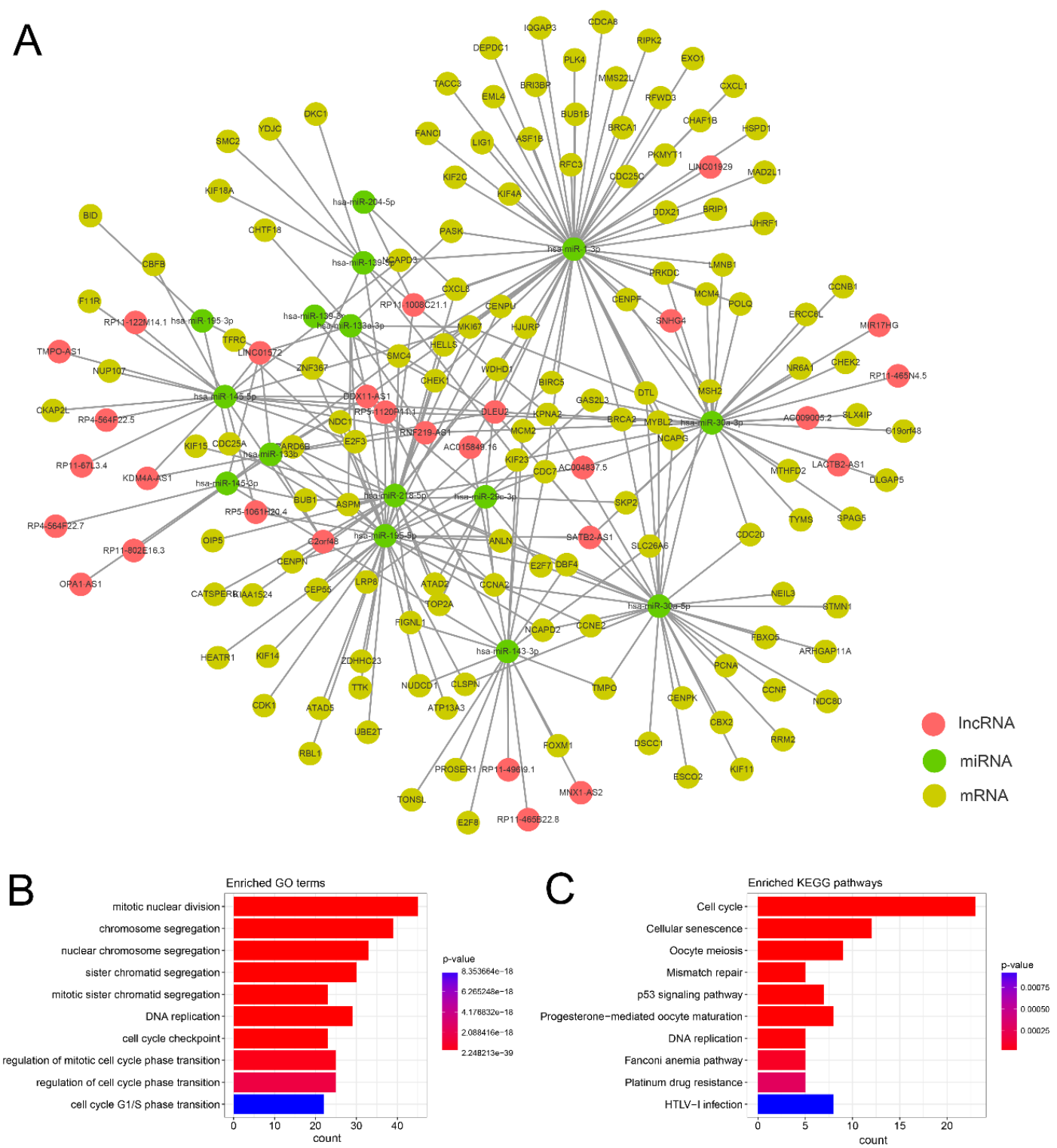

Figure 2. Global view and function analysis of the ceRNA network in GC. (A) Global view of the ceRNA network in GC. The lncRNA, mRNA, and miRNA were colored red, yellow, and green. There were 28 lncRNAs, 130 mRNAs, 16 miRNAs, and 173 edges in this network; (B) The top 10 significant gene ontology (GO) terms in GC-related ceRNA network; (C) The top 10 significant Kyoto Encyclopedia of Genes and Genomes (KEGG) pathways in GC-related ceRNA network. The count indicates the number of genes associated with a GO term in biological processes (BP) or a KEGG pathway.

\subsection{Topological Analysis of the lncRNA-Associated ceRNA Network in GC}

To clarify the roles of the dysregulated lncRNA-associated ceRNA network, we computed BC, which are topological features of ceRNA network, as shown in Table S4. A node with a higher BC plays a more important role in the ceRNA network, and is more likely to be associated with cancer $[19,41]$. Among the 174 nodes in the ceRNA network, the top 17 highest BC nodes (including 3 DELs, 3 DEMs, and 11 DEMis) were shown in Figure 3. We selected the three DELs (RP5-1120P11, DLEU2, and DDX11-AS1) with the highest BC values as the hub lncRNAs, and calculated the hub lncRNAs related to the first-neighbor DEMis, the second-neighbor DEMs, and the ceRNA triples. We determined that the hub lncRNAs were related to $87.5 \%$ of the DEMis, $87.69 \%$ of the DEMs, 
and $53.85 \%$ of the ceRNA triples in the ceRNA network (Table 1). These results indicated that the hub lncRNAs are potential key regulators controlling the ceRNA network in GC.

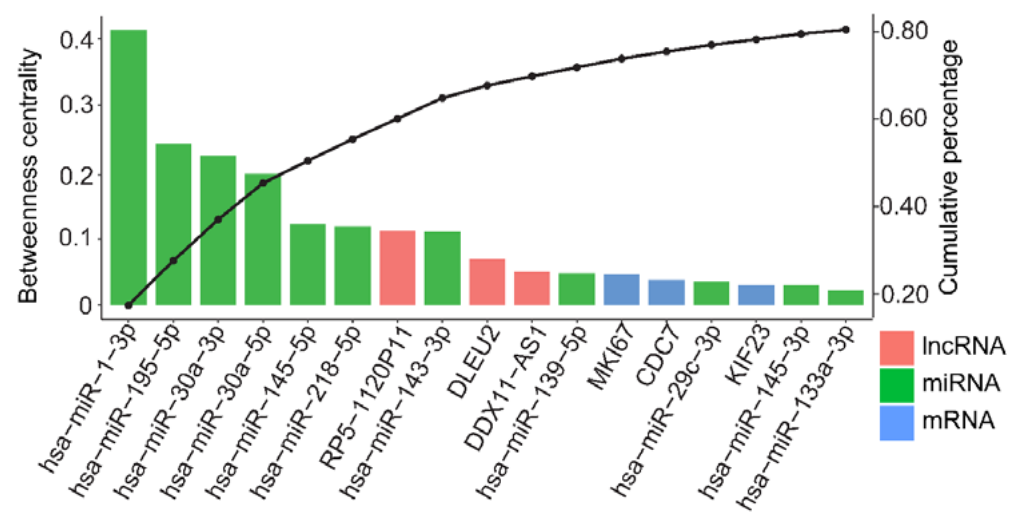

Figure 3. The top highest $17 \mathrm{BC}$ nodes in ceRNA network. The left $\mathrm{Y}$-axis denotes the betweenness centrality (BC) value of node, and the right Y-axis denotes the cumulative percentage of the BC. These nodes made up $80.5 \%$ of all the BCs in the ceRNA network.

Table 1. The number of hub lncRNA network-related DEMis, DEMs, and ceRNA triples.

\begin{tabular}{cccc}
\hline Associated Network & No. of DEMis & No. of DEMs & No. of Triples \\
\hline Three hub lncRNAs & $14(87.5 \%)$ & $114(87.69 \%)$ & $238(53.85 \%)$ \\
RP5-1120P11 & $9(56.25 \%)$ & $64(49.23 \%)$ & $92(20.81 \%)$ \\
DLEU2 & $7(43.75 \%)$ & $77(59.23 \%)$ & $96(21.72 \%)$ \\
DDX11-AS1 & $6(37.5 \%)$ & $45(34.62 \%)$ & $51(11.54 \%)$ \\
GC-related ceRNA & $16(100 \%)$ & $130(100 \%)$ & $442(100 \%)$ \\
\hline
\end{tabular}

\subsection{Reconstruction and Functional Analysis of Hub lncRNA-Associated Subnetworks in GC}

We assumed that the hub lncRNAs (RP5-1120P11, DLEU2, and DDX11-AS1) play critical roles in the GC-related ceRNA network. Accordingly, we reconstructed the hub IncRNA-associated subnetworks with the nodes, which were the hub lncRNAs related to the first-neighbor miRNAs and the second-neighbor mRNAs in the ceRNA network. To reveal the functions of the hub lncRNAs, we performed functional enrichment analysis for each associated subnetwork (Figure 4).

$R P 5-1120 P 11$ is the most important key regulator in the ceRNA network. RP5-1120P11 interacts with 9 DEMis (hsa-miR-139-3p, hsa-miR-139-5p, hsa-miR-143-3p, hsa-miR-145-5p, hsa-miR-195-3p, hsa-miR-195-5p, hsa-miR-218-5p, hsa-miR-29c-3p, and hsa-miR-30a-3p) and is co-regulated with 64 DEMs (Figure 4A). The function analysis of RP5-1120P11-associated subnetwork revealed $160 \mathrm{GO}$ terms in BP and 4 KEGG pathways (Figure 4D, Table S3). We determined that most of these GO terms and KEGG pathways are cell cycle process-related and cancer-related. However, there is no experimental evidence to support the function of RP5-1120P11 in recent studies.

DLEU2 is a lymphocytic leukemia-related gene and the host gene promoter for miR-15a/16-1 [42]. We observed that DLEU2 was co-regulated with 77 DEMs by acting as a ceRNA to sponge 6 DEMis (hsa-miR-1-3p, hsa-miR-30a-3p, hsa-miR-30a-5p, hsa-miR-133a-3p, hsa-miR-133b, and hsa-miR-145-5p) (Figure 4B). The functional analysis of DLEU2-associated subnetwork revealed $238 \mathrm{GO}$ terms in BP and 11 KEGG pathways (Figure 4E, Table S3). We determined that most of these GO terms are cell cycle process-related, and several pathways are cancer-related pathways, such as cell cycle, mismatch repair, p53 signaling pathway, Fanconi anemia pathway, and DNA replication. Intriguingly, DLEU2 downregulates hsa-miR-30-5p by ceRNA activity in previous study [43]. In the DLEU2-associated ceRNA network, hsa-miR-30a-5p is downregulated in GC, and negatively co-expressed with DLEU2 (Figure 5A,B). In addition, many studies have reported that hsa-miR-30a-5p is a tumor suppressor in GC $[44,45]$, and recent studies have shown that CCNA2, MYBL2, DTL, and STMN1 are regulated 
by hsa-miR-30a-5p [46-49]. In the network, we determined that CCNA2, MYBL2, DTL, and STMN1, which are significantly upregulated in GC (Figure 5A), are co-expressed with DLEU2 (Figure 5C) and negatively co-expressed with the same hsa-miR-30a-5p (Figure 5D). Therefore, we suspected that some ceRNA triples, such as DLEU2-hsa-miR-30a-5p-CCNA2, DLEU2-hsa-miR-30a-5p-MYBL2, DLEU2-hsa-miR-30a-5p-DTL, and DLEU2-hsa-miR-30a-5p-STMN1, are potential therapeutic targets in GC.

DDX11-AS1 is upregulated in multiple cancer types and affects sister chromatid cohesion [50]. The subnetwork related to DDX11-AS1 consisted of 6 DEMis and 45 DEMs (Figure 4C). The function analysis of the DDX11-AS1-associated subnetwork revealed 204 GO terms in BP and 8 KEGG pathways (Figure 4F, Table S3). We determined that most of these GO terms are cell cycle process-related and most pathways are cancer-related pathways, such as cell cycle, Fanconi anemia pathway, mismatch repair, DNA replication, and p53 signaling pathway.

All these results will provide us important cell cycle processes and cancer-related pathways information for understanding lncRNA-associated ceRNA network in GC.

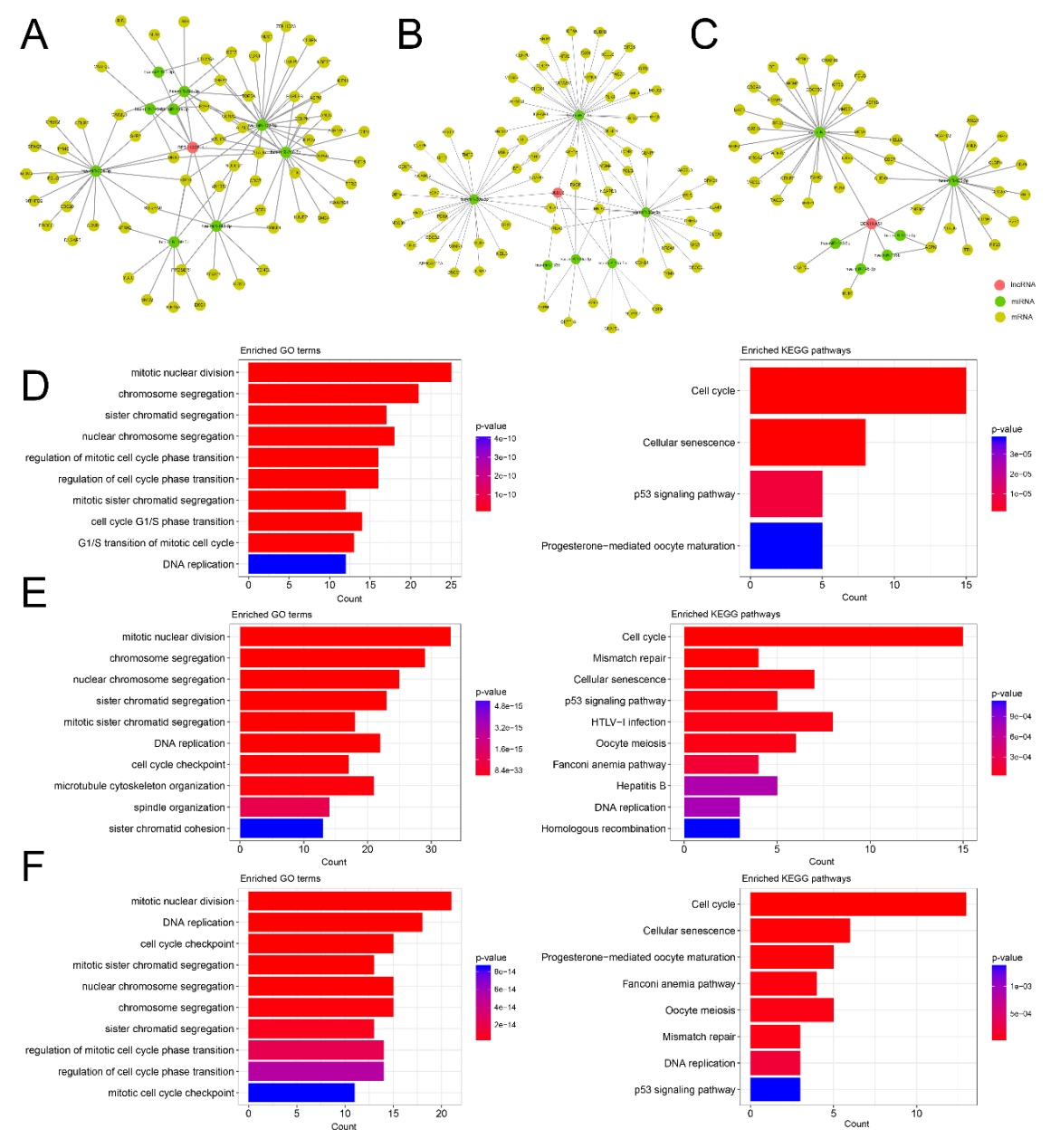

Figure 4. The ceRNA subnetworks and functional enrichment analysis of the hub lncRNAs. (A) The RP5-1120P11-associated ceRNA subnetwork; (B) DLEU2-associated ceRNA subnetwork; (C) DDX11-AS1-associated ceRNA subnetwork. The lncRNA, mRNA, and miRNA were colored red, yellow, and green; (D) The enrichment analysis result of RP5-1120P11-associated ceRNA subnetwork; (E) The enrichment analysis result of DLEU2-associated ceRNA subnetwork; (F) The enrichment analysis result of DDX11-AS1-associated ceRNA subnetwork. The count indicates the number of genes associated with a GO term in BP or a KEGG pathway. 

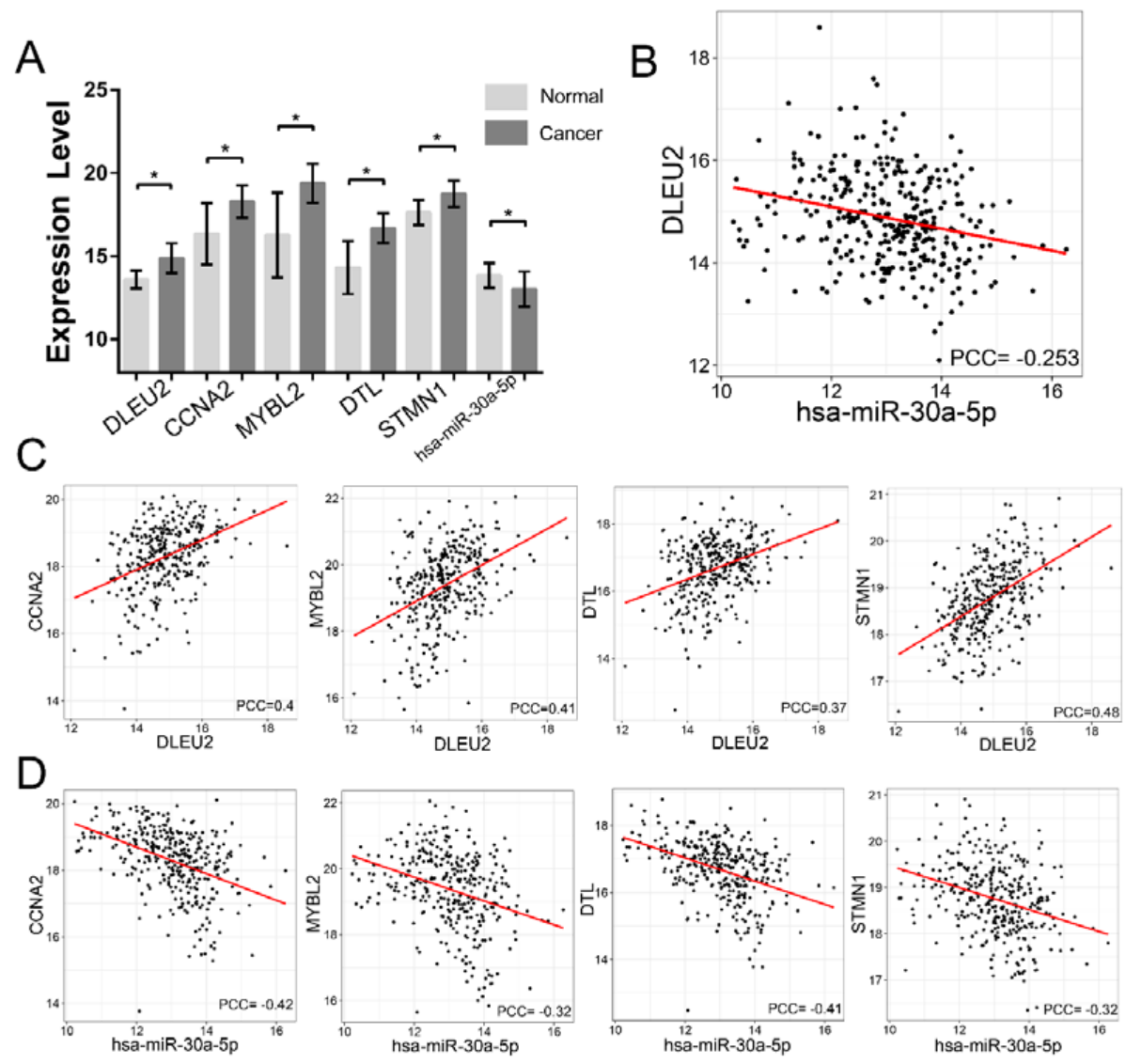

Figure 5. The expression levels and correlation of DLEU2 related DEMi and DEMs in GC. (A) The boxplot showed the expression levels of DLEU2 and DLEU2 related DEMs. * denotes $p<0.001$; (B) The scatter plots showed the correlation of expression between DLEU2 and hsa-miR-30-5p; (C) The scatter plots showed the correlation of expression between DLEU2 and DLEU2 related DEMs; (D) The scatter plots showed the correlation of expression between DLEU2 related DEMs and hsa-miR-30-5p. All the data were obtained from GDC.

\subsection{DLEU2 and DDX11-AS1 Are Upregulated in GC Tissues, Promote GC Cell Proliferation, and Inhibit miRNA Expression, Respectively}

To validate the findings, we first examined DLEU2 and DDX11-AS1 expression level in a cohort of 12 paired GC tissues, and compared with those of the adjacent normal tissues by using qRT-PCR. The DLEU2 and DDX11-AS1 expression levels were significantly upregulated in GC tissues (Figure 6A). siRNA-mediated silencing of lncRNAs were validated by qRT-PCR, and the knockdown efficiencies were achieved in BGC823 and MKN45 cell lines (Figure 6B). Next, we investigated the potential effect of DLEU2 and DDX11-AS1 in both cell lines. Knockdown of DLEU2 and DDX11-AS1 suppressed cell proliferation in BGC823 and MKN45 cells, as determined by CCK-8 assays (Figure 6B). The results of colony formation assay showed that BGC823 and MKN45 cells with DLEU2 and DDX11-AS1 knockdown formed significantly less colonies than control cells (Figure 6C). By miRNA target prediction, hsa-miR-30a-5p and hsa-miR-145-5p were noticed, as they have one putative target on DLEU2 and DDX11-AS1. DLEU2 and DDX11-AS1 complementarily matched the sequences on miRNA seed position, respectively. hsa-miR-30-5p regulated DLEU2 [43] and is a tumor suppressor in GC [44,45]. hsa-miR-145-5p inhibits gastric cancer cell migration and metastasis [51], and the relationship between DDX11-AS1 and hsa-miR-145-5p has not been reported in previous studies, but the correlation coefficient between DDX11-AS1 and hsa-miR-145-5p was the most significant (Table S2). Therefore, we selected hsa-miR-30-5p and hsa-miR-145-5p to verify the relationships between IncRNAs and miRNAs. By using qRT-PCR, we observed that knockdown of DLEU2 
and DDX11-AS1 significantly increased the hsa-miR-30-5p and hsa-miR-145-5p expression level, respectively (Figure 6D). These results indicated that DLEU2 and DDX11-AS1 might have oncogenic function and act as potential ceRNAs to sponge miRNAs in GC.

A

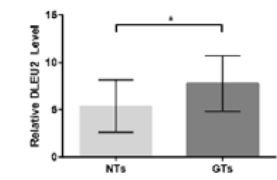

B

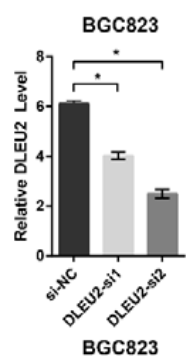

$1.0+{ }_{\text {SHAC }}^{\text {BGC823 }}$

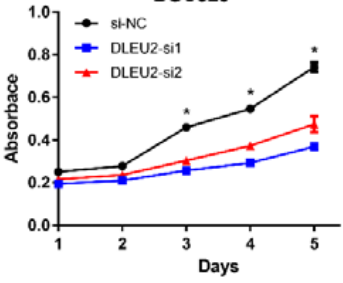

BGC823
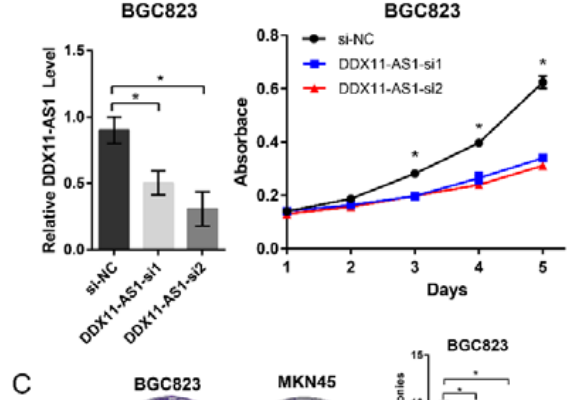

MKN45
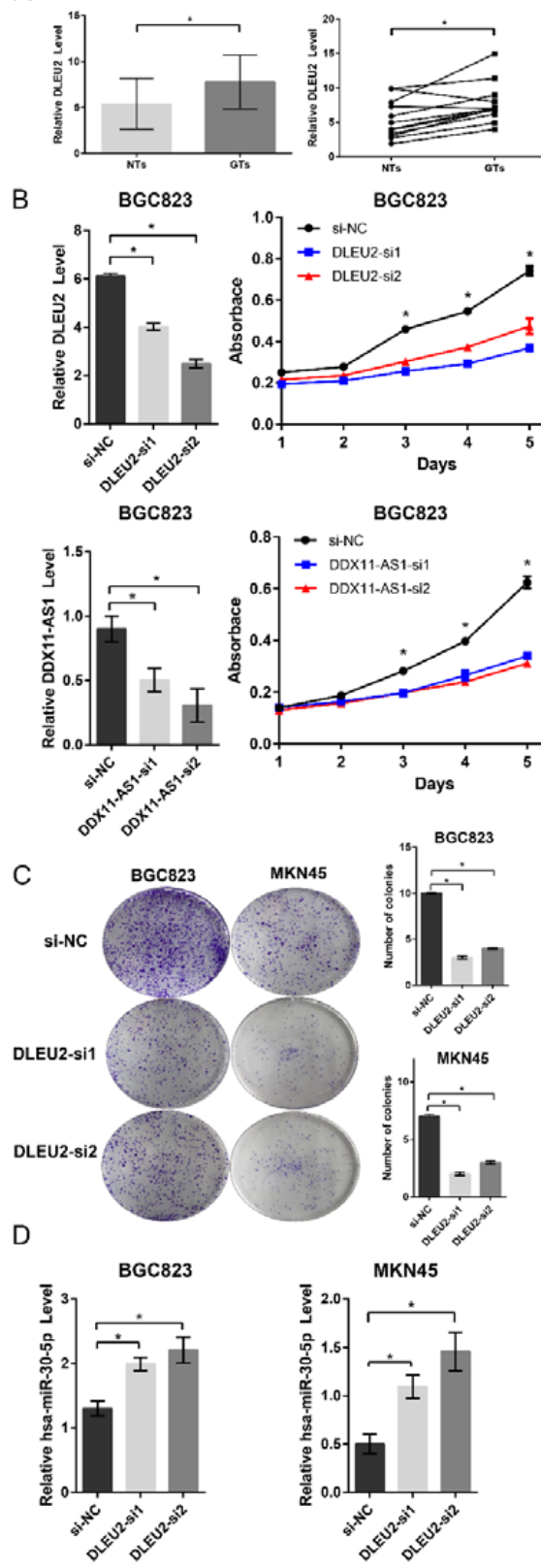
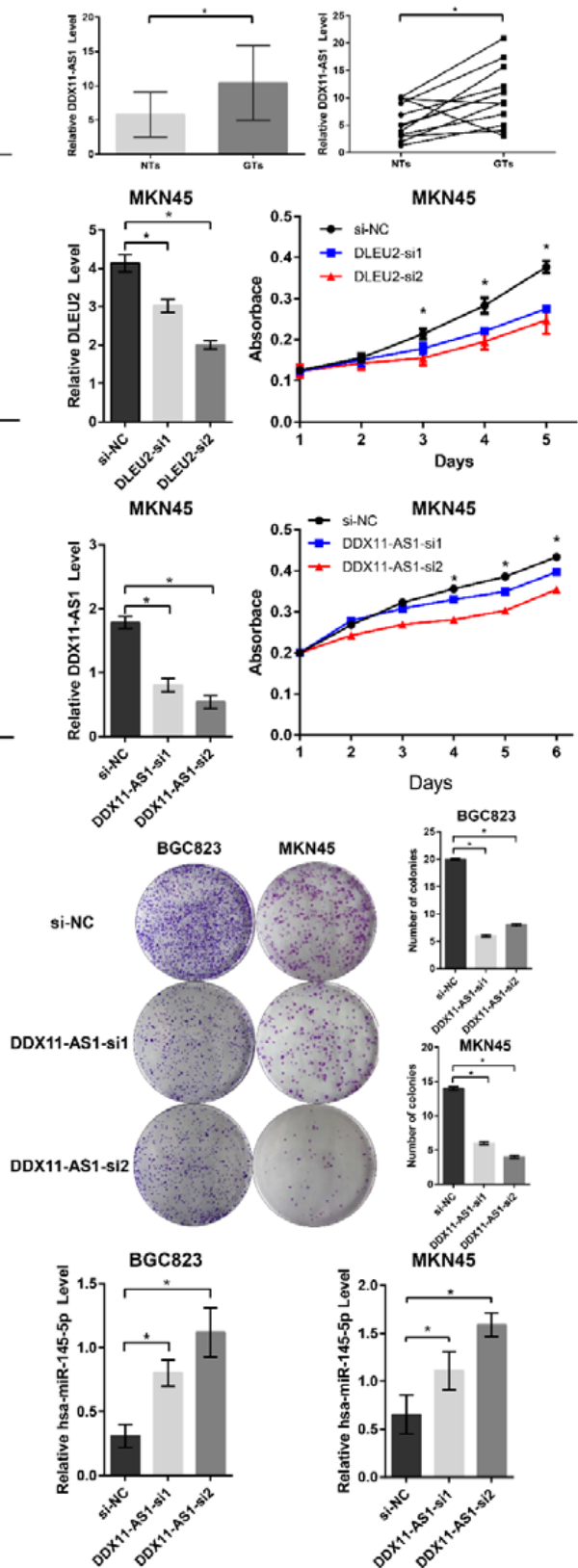

Figure 6. Functional validation of the predicted hub lncRNAs in GC. (A) The qRT-PCR results showed that the expression levels of DLEU2 and DDX11-AS1 in 12 pairs of GC tissues (GTs) were significantly higher than the adjacent normal tissues (NTs); (B) The qRT-PCR results showed DLEU2 and DDX11-AS1 knockdown in BGC823/MKN45 cells after transfection with NC (negative control), siRNA 1 (si-1), and siRNA 2 (si-2). The CCK-8 assays showed that knockdown of DLEU2 and DDX11-AS1 suppressed cell proliferation in BGC823/MKN45 cells; (C) The colony-forming assays showed that knockdown of DLEU2 and DDX11-AS1 suppressed cell proliferation in BGC823/MKN45 cells; (D) In BGC823 and MKN45 cells, knockdown of DLEU2 and DDX11-AS1 increased hsa-miR-30-5p and hsa-miR-145-5p expression, respectively. Biological experimental results were analyzed using paired or unpaired $t$-test, * denotes $p<0.05$. 


\section{Discussion}

Gastric Cancer is a leading cause of cancer-related death worldwide, and the second most common cancer in China. Many studies have demonstrated that coding RNAs and ncRNAs are closely related to GC, but the mechanism of GC remains to be elucidated. According to the ceRNA hypothesis, lncRNAs can act as miRNA sponges to construct a complex ceRNA network with miRNAs and mRNAs, as reported in many cancer types $[10,19,20,22,23,31]$. Thus ceRNAs play an important role in multilayered gene regulation and contribute to cancer development and pathology [52]. Some studies have constructed lncRNA-associated ceRNA networks in GC [23,24], but the mechanism of lncRNAs as ceRNAs in GC remains poorly explored.

In this study, we systematically integrated gene expression profiles and identified 571 DELs, 1097 DEMs, and 29 DEMis in GC. We determined that top DELs, such as FEZF1-AS1 [53], HOTAIR [54], HOXA11-AS [55], and HOTTIP [56], were GC-related lncRNAs, top DEMs such as CST1 [57], HOXC10 [58], HOXA13 [59], and HOXA11 [60] were GC-related coding RNAs, top DEMis such as hsa-miR-133b, hsa-miR-133a-3p [61], hsa-miR-1-3p [62], and hsa-miR-204-5p [63] were GC-related miRNAs. These differentially expressed genes were considered as potential key regulators in GC.

In this paper, we mainly focused on upregulated lncRNAs in GC samples based on ceRNA activity. Since the expression of lncRNAs are generally lower expressed than protein-coding genes [64], it is difficult to confirm the functions of the low expressed lncRNA in normal samples by experimental methods. On the other hand, the number of GC samples is much larger than controls. It is very difficult to compare a GC-related ceRNA network to a control ceRNA network. To construct the dysregulated ceRNA network in GC, we first identified miRNA-target interactions by integrating reliable online databases and prediction software. To reduce false positives, we used the PCC to filter the significantly co-expressed DEL-DEMi-DEM interactions. We then constructed a ceRNA network based on 442 potential ceRNA triples. The gene expression data and bioinformatics methods used differ from those employed in previous studies, the ceRNA triples were no overlap with others. After network topological analysis, we determined that the hub lncRNAs play crucial roles in GC. By reconstructing and functionally analyzing each hub lncRNA-associated subnetworks, we identified that three hub lncRNAs (RP5-1120P11, DLEU2, and DDX11-AS1) are involved in cell cycle related processes and cancer-related pathways. Although only a few studies of the three lncRNAs were available, some indirect evidence might support their potential regulatory function in GC. RP5-1120P11 might regulate many cancer-related signaling pathways and is associated with the poor prognosis in ovarian cancer [65]. DLEU2 is a lymphocytic leukemia-related gene and is the host gene promoter for miR-15a/16-1 [42], and acts as ceRNA to sponge miRNA in clear cell renal cell carcinoma (ccRCC) [43]. DDX11-AS1, which is upregulated in multiple cancer types, affects sister chromatid cohesion [50], and could play an important role in hepatocellular carcinoma (HCC) [66]. However, in previous studies, none of them has been reported as functional lncRNAs or act as ceRNAs in GC.

To validate our findings, experimental results revealed that DLEU2 and DDX11-AS1 might have oncogenic functions and act as potential ceRNAs to sponge miRNAs in GC. In the lncRNA-associated ceRNA network, hsa-miR-30a-5p is downregulated in GC, and negatively co-expressed with DLEU2 (Figure 5A,B). Cellular experimental results indicated that hsa-miR-30a-5p is inhibited by DLEU2 (Figure 6D). Intriguingly, DLEU2 downregulates hsa-miR-30-5p by ceRNA activity in cCRCC [43]. In addition, many studies have reported that hsa-miR-30a-5p is a tumor suppressor in GC [44,45], and recent studies have shown that CCNA2, MYBL2, DTL, and STMN1 are regulated by hsa-miR-30a-5p. CCNA2, which mediates cell proliferation and tumorigenesis [67], is regulated by miR-29a, miR-30a, and miR-141 to reactivate postnatal cardiomyocyte proliferation [46]. MYBL2, which is involved in tumorigenesis, has been implicated in cell proliferation and amplification. MYBL2 overexpression is associated with lower expression of miR-30a [47]. Overexpression of $D T L$, which plays a crucial role in tumor cell proliferation, is associated with poor survival rates and in GC [68]. DTL is regulated by hsa-miR-30a-5p to affect tumor growth in colon carcinoma [48]. STMN1, which is associated with poor prognosis in GC patients [69], is regulated by hsa-miR-30a in 
ovarian papillary serous carcinoma and ovarian clear cell carcinoma [49]. Therefore, we suspected that some ceRNA triples, such as DLEU2-hsa-miR-30a-5p-CCNA2, DLEU2-hsa-miR-30a-5p-MYBL2, DLEU2-hsa-miR-30a-5p-DTL, and DLEU2-hsa-miR-30a-5p-STMN1, are potential therapeutic targets in GC. Furthermore, hsa-miR-145-5p inhibit gastric cancer cell migration and metastasis [51], and we observed that knockdown of DDX11-AS1 significantly increased the hsa-miR-145-5p expression level, which means that DDX11-AS1 could act as potential ceRNA to sponge miRNA. However, the evidence of lncRNA inhibit miRNA expression, which we obtained by qRT-PCR, was indirect. Additional biological experiments will be required to further validate our findings.

These findings indicate that the hub lncRNA plays complex and critical roles in the development and pathology of GC. Although our study provides multistep computational and experimental methods to reveal functional lncRNAs in GC, biological experimental methods are still needed.

\section{Conclusions}

In summary, we employed multistep computational and experimental methods to reveal functional lncRNAs in GC based on the ceRNA hypothesis. By constructing and functionally analyzing lncRNA-associated network, we identified that three hub lncRNAs (RP5-1120P11, DLEU2, and DDX11-AS1) that are involved in cell cycle-related processes and cancer-related pathways. Furthermore, we confirmed that DLEU2 and DDX11-AS1 have oncogenic function and act as potential ceRNAs to sponge miRNAs. Our findings not only provide novel insights on ceRNA regulation in GC, but can also provide opportunities for the functional characterization of lncRNAs in future studies.

Supplementary Materials: The following are available online at http:/ /www.mdpi.com/2073-4425/9/6/303/s1, Figure S1: The strategy of integrative analysis of dysregulated lncRNA-associated ceRNA network in GC, Figure S2: PCC distribution in GC. (A) PCC higher the 95 percentile of the overall correlation distribution of lncRNA-mRNA pairs in GC. (B) PCC lower the 95 percentile of the overall correlation distribution of mRNA-miRNA pairs in GC. (C) PCC higher the 95 percentile of the overall correlation distribution of lncRNA-miRNA pairs in GC, Table S1: Differentially expressed lncRNAs, mRNAs, and miRNAs, Table S2: Potential ceRNA triples in GC-related ceRNA networks, Table S3: Functional enrichment analysis of ceRNA network. Table S4: The BC of all nodes in the ceRNA network.

Author Contributions: Y.L., D.F. and Y.N. conceived of and directed the project. H.L., Z.Z. and N.W. designed and performed the experiments. H.L. conducted the data analysis and interpreted the results. H.G. and H.Z. helped to design the study and review the data. H.L., Z.Z. and N.W. wrote and edited the manuscript. All authors have reviewed the manuscript and approved it for publication.

Funding: This research was funded by the National Natural Science Foundation of China (Grant No. $61471181,81702966,81730016,2017$ YFC0908300) and the Natural Science Foundation of Jilin Province (Grant No. 20140101194JC, 20150101056JC).

Conflicts of Interest: The authors declare no conflict of interest.

\section{References}

1. Van Cutsem, E.; Sagaert, X.; Topal, B.; Haustermans, K.; Prenen, H. Gastric cancer. Lancet 2016, 388, $2654-2664$. [CrossRef]

2. Ajani, J.A.; Lee, J.; Sano, T.; Janjigian, Y.Y.; Fan, D.; Song, S. Gastric adenocarcinoma. Nat. Rev. Dis. Primers 2017, 3, 17036. [CrossRef] [PubMed]

3. Torre, L.A.; Bray, F.; Siegel, R.L.; Ferlay, J.; Lortet-Tieulent, J.; Jemal, A. Global cancer statistics, 2012. CA Cancer J. Clin. 2015, 65, 87-108. [CrossRef] [PubMed]

4. Chen, W.; Zheng, R.; Zhang, S.; Zeng, H.; Zuo, T.; Xia, C.; Yang, Z.; He, J. Cancer incidence and mortality in China in 2013: An analysis based on urbanization level. Chin. J. Cancer Res. 2017, 29, 1-10. [CrossRef] [PubMed]

5. Dong, J.; Wang, R.; Ren, G.; Li, X.; Wang, J.; Sun, Y.; Liang, J.; Nie, Y.; Wu, K.; Feng, B.; et al. HMGA2-FOXL2 Axis Regulates Metastases and Epithelial-to-Mesenchymal Transition of Chemoresistant Gastric Cancer. Clin. Cancer Res. 2017, 23, 3461-3473. [CrossRef] [PubMed]

6. Bartel, D.P. MicroRNAs: Target recognition and regulatory functions. Cell 2009, 136, 215-233. [CrossRef] [PubMed] 
7. Lin, S.; Gregory, R.I. MicroRNA biogenesis pathways in cancer. Nat. Rev. Cancer 2015, 15, 321-333. [CrossRef] [PubMed]

8. Engreitz, J.M.; Ollikainen, N.; Guttman, M. Long non-coding RNAs: Spatial amplifiers that control nuclear structure and gene expression. Nat. Rev. Mol. Cell Biol. 2016, 17, 756-770. [CrossRef] [PubMed]

9. Schmitt, A.M.; Chang, H.Y. Long noncoding RNAs in cancer pathways. Cancer Cell 2016, 29, $452-463$. [CrossRef] [PubMed]

10. Du, Z.; Sun, T.; Hacisuleyman, E.; Fei, T.; Wang, X.; Brown, M.; Rinn, J.L.; Lee, M.G.; Chen, Y.; Kantoff, P.W.; et al. Integrative analyses reveal a long noncoding RNA-mediated sponge regulatory network in prostate cancer. Nat. Commun. 2016, 7, 10982. [CrossRef] [PubMed]

11. Thomson, D.W.; Dinger, M.E. Endogenous microRNA sponges: Evidence and controversy. Nat. Rev. Genet. 2016, 17, 272-283. [CrossRef] [PubMed]

12. Yvonne, T.; John, R.; Pier Paolo, P. The multilayered complexity of ceRNA crosstalk and competition. Nature 2014, 505, 344-352.

13. Poliseno, L.; Salmena, L.; Zhang, J.; Carver, B.; Haveman, W.J.; Pandolfi, P.P. A coding-independent function of gene and pseudogene mRNAs regulates tumour biology. Nature 2010, 465, 1033-1038. [CrossRef] [PubMed]

14. Karreth, F.A.; Reschke, M.; Ruocco, A.; Ng, C.; Chapuy, B.; Léopold, V.; Sjoberg, M.; Keane, T.M.; Verma, A.; Ala, U. The BRAF pseudogene functions as a competitive endogenous RNA and induces lymphoma in vivo. Cell 2015, 161, 319-332. [CrossRef] [PubMed]

15. Liu, X.-H.; Sun, M.; Nie, F.-Q.; Ge, Y.-B.; Zhang, E.-B.; Yin, D.-D.; Kong, R.; Xia, R.; Lu, K.-H.; Li, J.-H.; et al. Lnc RNA HOTAIR functions as a competing endogenous RNA to regulate HER2 expression by sponging miR-331-3p in gastric cancer. Mol. Cancer 2014, 13, 92. [CrossRef] [PubMed]

16. Hu, Y.; Wang, J.; Qian, J.; Kong, X.; Tang, J.; Wang, Y.; Chen, H.; Hong, J.; Zou, W.; Chen, Y.; et al. Long noncoding RNA GAPLINC regulates CD44-dependent cell invasiveness and associates with poor prognosis of gastric cancer. Cancer Res. 2014, 74, 6890-6902. [CrossRef] [PubMed]

17. Song, Y.X.; Sun, J.X.; Zhao, J.H.; Yang, Y.C.; Shi, J.X.; Wu, Z.H.; Chen, X.W.; Gao, P.; Miao, Z.F.; Wang, Z.N. Non-coding RNAs participate in the regulatory network of CLDN4 via ceRNA mediated miRNA evasion. Nat. Commun. 2017, 8, 289. [CrossRef] [PubMed]

18. Peng, W.; Si, S.; Zhang, Q.; Li, C.; Zhao, F.; Wang, F.; Yu, J.; Ma, R. Long non-coding RNA MEG3 functions as a competing endogenous RNA to regulate gastric cancer progression. J. Exp. Clin. Cancer Res. 2015, 34, 79. [CrossRef] [PubMed]

19. Zhou, M.; Wang, X.; Shi, H.; Cheng, L.; Wang, Z.; Zhao, H.; Yang, L.; Sun, J. Characterization of long non-coding RNA-associated ceRNA network to reveal potential prognostic lncRNA biomarkers in human ovarian cancer. Oncotarget 2016, 7, 12598-12611. [CrossRef] [PubMed]

20. Cao, Y.; Wang, P.; Ning, S.; Xiao, W.; Xiao, B.; Li, X. Identification of prognostic biomarkers in glioblastoma using a long non-coding RNA-mediated, competitive endogenous RNA network. Oncotarget 2016, 7, 41737-41747. [CrossRef] [PubMed]

21. Lu, M.; Xu, X.; Xi, B.; Dai, Q.; Li, C.; Su, L.; Zhou, X.; Tang, M.; Yao, Y.; Yang, J. Molecular Network-Based Identification of Competing Endogenous RNAs in Thyroid Carcinoma. Genes 2018, 9, 44. [CrossRef] [PubMed]

22. Zhang, J.; Le, T.D.; Liu, L.; Li, J. Inferring miRNA sponge co-regulation of protein-protein interactions in human breast cancer. BMC Bioinf. 2017, 18, 243. [CrossRef] [PubMed]

23. Xia, T.; Liao, Q.; Jiang, X.; Shao, Y.; Xiao, B.; Xi, Y.; Guo, J. Long noncoding RNA associated-competing endogenous RNAs in gastric cancer. Sci. Rep. 2014, 4, 6088. [CrossRef] [PubMed]

24. Li, C.-Y.; Liang, G.-Y.; Yao, W.-Z.; Sui, J.; Shen, X.; Zhang, Y.-Q.; Peng, H.; Hong, W.-W.; Ye, Y.-C.; Zhang, Z.-Y.; et al. Integrated analysis of long non-coding RNA competing interactions reveals the potential role in progression of human gastric cancer. Int. J. Oncol. 2016, 48, 1965-1976. [CrossRef] [PubMed]

25. The Cancer Genome Atlas Research Network. Comprehensive molecular characterization of gastric adenocarcinoma. Nature 2014, 513, 202-209.

26. Harrow, J.; Frankish, A.; Gonzalez, J.M.; Tapanari, E.; Diekhans, M.; Kokocinski, F.; Aken, B.L.; Barrell, D.; Zadissa, A.; Searle, S.; et al. GENCODE: The reference human genome annotation for The ENCODE Project. Genome Res. 2012, 22, 1760. [CrossRef] [PubMed] 
27. Agarwal, V.; Bell, G.W.; Nam, J.W.; Bartel, D.P. Predicting effective microRNA target sites in mammalian mRNAs. Elife 2015, 4, e05005. [CrossRef] [PubMed]

28. Dweep, H.; Gretz, N. miRWalk2.0: A comprehensive atlas of microRNA-target interactions. Nat. Methods 2015, 12, 697. [CrossRef] [PubMed]

29. Chou, C.-H.; Chang, N.-W.; Shrestha, S.; Hsu, S.-D.; Lin, Y.-L.; Lee, W.-H.; Yang, C.-D.; Hong, H.-C.; Wei, T.-Y.; $\mathrm{Tu}$, S.-J.; et al. miRTarBase 2016: Updates to the experimentally validated miRNA-target interactions database. Nucleic Acids Res. 2015, 44, D239-D247. [CrossRef] [PubMed]

30. Vlachos, I.S.; Paraskevopoulou, M.D.; Karagkouni, D.; Georgakilas, G.; Vergoulis, T.; Kanellos, I.; Anastasopoulos, I.-L.; Maniou, S.; Karathanou, K.; Kalfakakou, D.; et al. DIANA-TarBase v7. 0: Indexing more than half a million experimentally supported miRNA: MRNA interactions. Nucleic Acids Res. 2014, 43, D153-D159. [CrossRef] [PubMed]

31. Wang, P.; Ning, S.; Zhang, Y.; Li, R.; Ye, J.; Zhao, Z.; Zhi, H.; Wang, T.; Guo, Z.; Li, X. Identification of lncRNA-associated competing triplets reveals global patterns and prognostic markers for cancer. Nucleic Acids Res. 2015, 43, 3478-3489. [CrossRef] [PubMed]

32. Shannon, P.; Markiel, A.; Ozier, O.; Baliga, N.S.; Wang, J.T.; Ramage, D.; Amin, N.; Schwikowski, B.; Ideker, T. Cytoscape: A software environment for integrated models of biomolecular interaction networks. Genome Res. 2003, 13, 2498-2504. [CrossRef] [PubMed]

33. Yu, G.; Wang, L.G.; Han, Y.; He, Q.Y. clusterProfiler: An R Package for Comparing Biological Themes among Gene Clusters. OMICS J. Integr. Biol. 2012, 16, 284-287. [CrossRef] [PubMed]

34. The Gene Ontology Consortium. Expansion of the Gene Ontology knowledgebase and resources. Nucleic Acids Res. 2017, 45, D331-D338. [CrossRef]

35. Kanehisa, M.; Furumichi, M.; Tanabe, M.; Sato, Y.; Morishima, K. KEGG: New perspectives on genomes, pathways, diseases and drugs. Nucleic Acids Res. 2017, 45, D353-D361. [CrossRef] [PubMed]

36. Otto, T.; Sicinski, P. Cell cycle proteins as promising targets in cancer therapy. Nat. Rev. Cancer 2017, 17, 93-115. [CrossRef] [PubMed]

37. Whibley, C.; Pharoah, P.D.P.; Hollstein, M. p53 polymorphisms: Cancer implications. Nat. Rev. Cancer 2009, 9, 95-107. [CrossRef] [PubMed]

38. Ceccaldi, R.; Sarangi, P.; D'Andrea, A.D. The Fanconi anaemia pathway: New players and new functions. Nat. Rev. Mol. Cell Biol. 2016, 17, 337-349. [CrossRef] [PubMed]

39. Helleday, T.; Petermann, E.; Lundin, C.; Hodgson, B.; Sharma, R.A. DNA repair pathways as target for cancer therapy. Nat. Rev. Cancer 2008, 8, 193-204. [CrossRef] [PubMed]

40. Gottesman, M.M.; Lavi, O.; Hall, M.D.; Gillet, J.P. Toward a Better Understanding of the Complexity of Cancer Drug Resistance. Annu. Rev. Pharmacol. Toxicol. 2016, 56, 85-102. [CrossRef] [PubMed]

41. Song, C.; Zhang, J.; Liu, Y.; Pan, H.; Qi, H.P.; Cao, Y.G.; Zhao, J.M.; Li, S.; Guo, J.; Sun, H.L.; et al. Construction and analysis of cardiac hypertrophy-associated lncRNA-mRNA network based on competitive endogenous RNA reveal functional lncRNAs in cardiac hypertrophy. Oncotarget 2016, 7, 10827-10840. [CrossRef] [PubMed]

42. Angela, G.; Nupur, B.; Rainer, C.; Melanie, R.; Cordula, T.; Katharina, F.; Irina, I.; Manuela, Z.; Maïwen, C.H.; Christopher, O.; et al. Epigenetic upregulation of lncRNAs at 13q14.3 in leukemia is linked to the In Cis downregulation of a gene cluster that targets NF-kB. PLoS Genet. 2013, 9, e1003373.

43. Chen, Z.; Zhang, J.; Zhang, Z.; Feng, Z.; Wei, J.; Lu, J.; Yong, F.; Liang, Y.; Cen, J.; Pan, Y.; et al. The putative tumor suppressor microRNA-30a-5p modulates clear cell renal cell carcinoma aggressiveness through repression of ZEB2. Cell Death Dis. 2017, 8, e2859. [CrossRef] [PubMed]

44. Sousa, J.F.; Nam, K.T.; Petersen, C.P.; Lee, H.-J.; Yang, H.-K.; Kim, W.H.; Goldenring, J.R. miR-30-HNF4 $\gamma$ and miR-194-NR2F2 regulatory networks contribute to the up-regulation of metaplasia markers in the stomach. Gut 2016, 65, 914-924. [CrossRef] [PubMed]

45. Liu, X.; Ji, Q.; Zhang, C.; Liu, X.; Liu, Y.; Liu, N.; Sui, H.; Zhou, L.; Wang, S.; Li, Q. miR-30a acts as a tumor suppressor by double-targeting COX-2 and BCL9 in H. pylori gastric cancer models. Sci. Rep. 2017, 7, 7113. [CrossRef] [PubMed]

46. Zhang, Y.; Matsushita, N.; Eigler, T.; Marban, E. Targeted MicroRNA Interference Promotes Postnatal Cardiac Cell Cycle Re-Entry. J. Regen. Med. 2013, 2, 2. [CrossRef] [PubMed] 
47. Fuster, O.; Llop, M.; Dolz, S.; García, P.; Such, E.; Ibáñez, M.; Luna, I.; Gómez, I.; López, M.; Cervera, J.; et al. Adverse prognostic value of MYBL2 overexpression and association with microRNA-30 family in acute myeloid leukemia patients. Leuk. Res. 2013, 37, 1690-1696. [CrossRef] [PubMed]

48. Baraniskin, A.; Birkenkampdemtroder, K.; Maghnouj, A.; Zöllner, H.; Munding, J.; Kleinscory, S.; Reinacherschick, A.; Schwartewaldhoff, I.; Schmiegel, W.; Hahn, S.A. MiR-30a-5p suppresses tumor growth in colon carcinoma by targeting DTL. Carcinogenesis 2012, 33, 732-739. [CrossRef] [PubMed]

49. Zhao, H.; Ding, Y.; Tie, B.; Sun, Z.F.; Jiang, J.Y.; Zhao, J.; Lin, X.; Cui, S. miRNA expression pattern associated with prognosis in elderly patients with advanced OPSC and OCC. Int. J. Oncol. 2013, 43, 839-849. [CrossRef] [PubMed]

50. Marchese, F.P.; Grossi, E.; Marín-Béjar, O.; Bharti, S.K.; Raimondi, I.; González, J.; Martínez-Herrera, D.J.; Athie, A.; Amadoz, A.; Brosh, R.M., Jr.; et al. A Long Noncoding RNA Regulates Sister Chromatid Cohesion. Mol. Cell 2016, 63, 397-407. [CrossRef] [PubMed]

51. Lei, C.; Du, F.; Sun, L.; Li, T.; Li, T.; Min, Y.; Nie, A.; Wang, X.; Geng, L.; Lu, Y.; et al. miR-143 and miR-145 inhibit gastric cancer cell migration and metastasis by suppressing MYO6. Cell Death Dis. 2017, 8, e3101. [CrossRef] [PubMed]

52. Lü, M.; Tang, B.; Zeng, S.; Hu, C.; Xie, R.; Wu, Y.; Wang, S.; He, F.; Yang, S. Expansion of the Gene Ontology knowledgebase and resources. Oncogene 2015, 35, 3524-3534. [CrossRef] [PubMed]

53. Liu, Y.W.; Xia, R.; Lu, K.; Xie, M.; Yang, F.; Sun, M.; De, W.; Wang, C.; Ji, G. LincRNAFEZF1-AS1 represses p21 expression to promote gastric cancer proliferation through LSD1-Mediated H3K4me2 demethylation. Mol. Cancer 2017, 16, 39. [CrossRef] [PubMed]

54. Gu, J.; Li, Y.; Fan, L.; Zhao, Q.; Tan, B.; Hua, K.; Wu, G. Identification of aberrantly expressed long non-coding RNAs in stomach adenocarcinoma. Oncotarget 2017, 8, 49201-49216. [CrossRef] [PubMed]

55. Sun, M.; Nie, F.; Wang, Y.; Zhang, Z.; Hou, J.; He, D.; Xie, M.; Xu, L.; De, W.; Wang, Z.; et al. LncRNA HOXA11-AS Promotes Proliferation and Invasion of Gastric Cancer by Scaffolding the Chromatin Modification Factors PRC2, LSD1, and DNMT1. Cancer Res. 2016, 76, 6299-6310. [CrossRef] [PubMed]

56. Wang, S.S.; Wuputra, K.; Liu, C.J.; Lin, Y.C.; Chen, Y.T.; Chai, C.Y.; Lin, C.S.; Kuo, K.K.; Tsai, M.H.; Wang, S.W.; et al. Oncogenic function of the homeobox A13-long noncoding RNA HOTTIP-insulin growth factor-binding protein 3 axis in human gastric cancer. Oncotarget 2016, 7, 36049-36064. [CrossRef] [PubMed]

57. Choi, E.H.; Kim, J.T.; Kim, J.H.; Kim, S.Y.; Song, E.Y.; Kim, J.W.; Kim, S.Y.; Yeom, Y.I.; Kim, I.H.; Lee, H.G. Upregulation of the cysteine protease inhibitor, cystatin $\mathrm{SN}$, contributes to cell proliferation and cathepsin inhibition in gastric cancer. Clin. Chim. Acta 2009, 406, 45-51. [CrossRef] [PubMed]

58. Zheng, J.; Ge, P.; Liu, X.; Wei, J.; Wu, G.; Li, X. MiR-136 inhibits gastric cancer-specific peritoneal metastasis by targeting HOXC10. Tumour Biol. J. Int. Soc. Oncodev. Biol. Med. 2017, 39. [CrossRef] [PubMed]

59. Han, Y.; Tu, W.-W.; Wen, Y.-G.; Li, D.-P.; Qiu, G.-Q.; Tang, H.-M.; Peng, Z.-H.; Zhou, C.-Z. Identification and validation that up-expression of HOXA13 is a novel independent prognostic marker of a worse outcome in gastric cancer based on immunohistochemistry. Med. Oncol. 2013, 30, 564. [CrossRef] [PubMed]

60. Cui, Y.; Gao, D.; Linghu, E.; Zhan, Q.; Chen, R.; Brock, M.V.; Herman, J.G.; Guo, M. Epigenetic changes and functional study of HOXA11 in human gastric cancer. Epigenomics 2015, 7, 201-213. [CrossRef] [PubMed]

61. Liu, Y.; Zhang, X.; Zhang, Y.; Hu, Z.; Yang, D.; Wang, C.; Guo, M.; Cai, Q. Identification of miRNomes in human stomach and gastric carcinoma reveals miR-133b/a-3p as therapeutic target for gastric cancer. Cancer Lett. 2015, 369, 58-66. [CrossRef] [PubMed]

62. Xie, M.; Dart, D.A.; Guo, T.; Xing, X.F.; Cheng, X.J.; Du, H.; Jiang, W.G.; Wen, X.Z.; Ji, J.F. MicroRNA-1 acts as a tumor suppressor microRNA by inhibiting angiogenesis-related growth factors in human gastric cancer. Gastric Cancer 2018, 21, 41-54. [CrossRef] [PubMed]

63. Canu, V.; Sacconi, A.; Lorenzon, L.; Biagioni, F.; Lo, S.F.; Diodoro, M.G.; Muti, P.; Garofalo, A.; Strano, S.; D'Errico, A.; et al. MiR-204 down-regulation elicited perturbation of a gene target signature common to human cholangiocarcinoma and gastric cancer. Oncotarget 2017, 8, 29540-29557. [CrossRef] [PubMed]

64. Derrien, T.; Johnson, R.; Bussotti, G.; Tanzer, A.; Djebali, S.; Tilgner, H.; Guernec, G.; Martin, D.; Merkel, A.; Knowles, D.G.; et al. The GENCODE v7 catalog of human long noncoding RNAs: Analysis of their gene structure, evolution, and expression. Genome Res. 2012, 22, 1775-1789. [CrossRef] [PubMed]

65. Fang, L.; Wang, H.; Li, P. Systematic analysis reveals a lncRNA-mRNA co-expression network associated with platinum resistance in high-grade serous ovarian cancer. Investig. New Drugs 2018, 36, 187-194. [CrossRef] [PubMed] 
66. Min, S.; Zhang, X.Y.; Yu, H.; Xiang, S.H.; Ling, X.; Wei, J.; Wu, Q.; Jia, R.; Wang, Y.G.; Lu, X.J. DDX11-AS1 as potential therapy targets for human hepatocellular carcinoma. Oncotarget 2017, 8, 44195-44202.

67. Gopinathan, L.; Tan, S.L.; Padmakumar, V.C.; Coppola, V.; Tessarollo, L.; Kaldis, P. Loss of Cdk2 and cyclin A2 impairs cell proliferation and tumorigenesis. Cancer Res. 2014, 74, 3870. [CrossRef] [PubMed]

68. Kobayashi, H.; Komatsu, S.; Ichikawa, D.; Kawaguchi, T.; Hirajima, S.; Miyamae, M.; Okajima, W.; Ohashi, T.; Kosuga, T.; Konishi, H.; et al. Overexpression of denticleless E3 ubiquitin protein ligase homolog (DTL) is related to poor outcome in gastric carcinoma. Oncotarget 2015, 6, 36615-36624. [CrossRef] [PubMed]

69. Bai, T.; Yokobori, T.; Altan, B.; Ide, M.; Mochiki, E.; Yanai, M.; Kimura, A.; Kogure, N.; Yanoma, T.; Suzuki, M.; et al. High STMN1 level is associated with chemo-resistance and poor prognosis in gastric cancer patients. Br. J. Cancer 2017, 1177-1185. [CrossRef] [PubMed]

(C) 2018 by the authors. Licensee MDPI, Basel, Switzerland. This article is an open access article distributed under the terms and conditions of the Creative Commons Attribution (CC BY) license (http:/ / creativecommons.org/licenses/by/4.0/). 